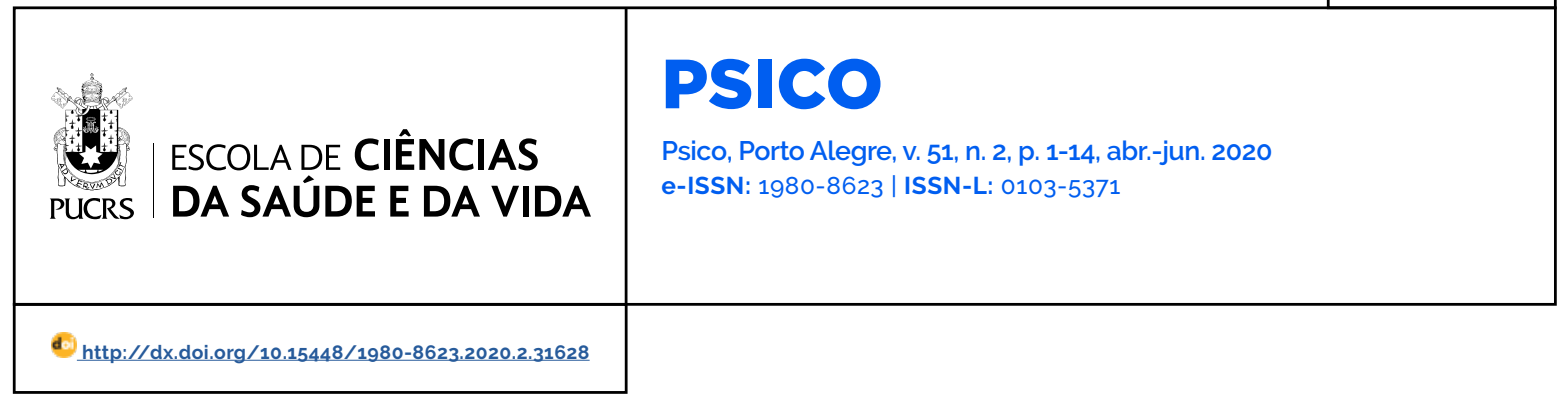

SEÇÃO: ARTIGO

\title{
Propriedades psicométricas da escala de presença em tecnologias ubíquas
}

\author{
Psychometric properties of presence on ubiquitous technologies' scale \\ Propiedades psicométricas de la escala de presencia en tecnologías ubicuas
}

\section{Fabiane Friedrich \\ Schütz ${ }^{1}$ \\ orcid.org/0000-0002-2110-4576 \\ fabianefschutz@gmail.com}

\section{Livia Maria Bedin ${ }^{1}$ \\ orcid.org/0000-0002-7472-2255 \\ liviabedin@gmail.com}

\author{
Jorge Castellá Sarriera ${ }^{1}$ \\ orcid.org/0000-0001-8297-4957 \\ jorgesarriera@gmail.com
}

Recebido em: 8 ago. 2018 Aprovado em: 26 fev. 2020. Publicado em: 4 set. 2020

\section{(c) (1)}

Artigo está licenciado sob forma de uma licença Creative Commons Atribuição 4.0 Internacional.
Resumo: Este estudo teve como objetivo desenvolver uma escala de presença nas tecnologias ubíquas. O processo de desenvolvimento do instrumento teve como etapas 1) a escolha dos atributos comportamentais referentes ao objeto de estudo e à operacionalização do construto, 2) a apresentação a três juízes, dois grupos focais, 3) uma aplicação-piloto em uma amostra reduzida $(n=30)$ e, por fim, 4) uma aplicação com a 458 participantes. Foram realizadas análises de consistência interna, análise fatorial confirmatória e análise fatorial confirmatória multigrupo. A versão final da "Escala de Presença em Tecnologias Ubiquas (EPTU)" conta com 14 itens, agrupados em três fatores independentes (autopresença, presença espacial e presença social), e apresenta um bom indice de consistência interna ( $\alpha=$ 0,905 ) e índices de ajuste aceitáveis ( $\chi 2=116,83 ; g l=74 ; p<0,01 ; C F I=0,99 ; R M S E A$ $=0,035 ; S R M R=0,053$ ), com parâmetros psicométricos equivalentes por gênero.

Palavras-chave: autopresença, presença espacial, presença social, tecnologias ubíquas, avaliação psicológica.

Abstract: This study aimed to develop a scale of presence in ubiquitous technologies. The development process of the instrument had 4 stages: 1) the choice of behavioral attributes related to the object of study and the operation of the construct, 2) the presentation to three judges, two focus groups and a 3) pilot application in a reduced sample $(n=30)$ and 4 ) an application with 458 participants. In order to obtain validity and reliability evidences we performed internal consistency analyzes, confirmatory factor analysis and multigroup confirmatory factorial analysis. The final version of the Presence Scale in Ubiquitous Technologies has 14 items, grouped into three independent factors (Self-Presence, Spatial Presence and Social Presence), and has a good internal consistency index (alpha $=0.905$ ) and an acceptable adjustment index $(\chi 2=116.83, g l=74, p<0.01, C F I=0.99, R M-$ $S E A=0.035, S R M R=0.053$ ), with equivalent psychometric parameters by gender. Keywords: self-presence, spatial presence, social presence, ubiquitous technologies, psychological assessment.

Resumen: Este estudio tuvo como objetivo el desarrollo de una escala de presencia en las tecnologías ubicuas. El proceso de desarrollo del instrumento tuvo como etapas 1) la elección de los atributos comportamentales referentes al objeto de estudio y la operacionalización del constructo, 2) la presentación a tres jueces, dos grupos focales, 3) una aplicación piloto en una muestra reducida $(n=30)$, y por último, 4) una aplicación con 458 participantes. Se realizaron análisis de consistencia interna, análisis factorial confirmatorio y análisis factorial confirmatorio multigrupo. La versión final de la Escala de Presencia en Tecnologias Ubíquicas cuenta con 14 items, agrupados en tres factores independientes (Autopresencia, Presencia Espacial y Presencia Social), y presenta un buen indice de consistencia interna (alfa $=0,905)$ y un indice de ajuste aceptable $(\chi 2=116,83 ; g l=74 ; p<0,01 ; C F I=0,99 ; R M S E A=0,035$; $S R M R=0,053$ ) con parámetros psicométricos equivalentes por género Palabras-clave: autopresencia, presencia espacial, presencia social, tecnologias ubicuas, evaluación psicológica. 
A ideia de que as tecnologias, especialmente as ubiquas, impactam no cotidiano têm se tornado um tópico de interesse para a psicologia. O fenômeno da presença na interação com tecnologias vem sendo estudado desde a década de 1970 quando Short, Williams e Christie (1976) apresentaram uma definição para a comunicação mediada por tecnologia como uma qualidade do meio que implica no grau de percepção do outro na interação e na consequência dessa nos relacionamentos interpessoais. No início dos anos 2000, a International Society for Presence Research definiu essa experiência como:

um estado psicológico no qual ainda que
parte ou a totalidade da experiência de
um individuo seja gerada por e/ou filtra-
da por meio de tecnologia produzida por
humanos, parte ou a totalidade da per-
cepção do individuo falha em reconhecer
precisamente o papel da tecnologia na
experiência (Lombard \& Jones, 2015).

Para fins deste estudo, assume-se que a presença é uma reação ao mundo externo, ou ao que se percebe como mundo externo, podendo ocorrer na ausência ou não de tecnologias (Heeter, 1992). Compreende-se que se trata de um fenômeno derivado de uma propriedade subjetiva, experiencial, no âmbito do estado psicológico (Felton, 2014), podendo ser despertada por estímulos internos e externos (Biocca, Harms, \& Burgoon, 2003), por meio da percepção de que não há mediação na experiência mediada (Lee, 2004). Especificamente, compreende-se que o fenômeno de presença é multidimensional e são estudadas três dimensões principais: presença social, presença espacial e autopresença.

A presença espacial é compreendida como a percepção de estar em outro local, fisicamente, juntamente com uma mudança de foco de atenção direcionada a esse outro espaço (Hartmann et al., 2015). Opera como uma ferramenta evolutiva, que pode ser usada para rastrear as diferenças entre as sensações imaginadas e as elicitadas pelo mundo sensorial (Riva, Wiederhold, \& Mantovani, 2019). Para haver presença espacial é necessário que haja a sensação de estar fisicamente situado no ambiente espacial e a percepção de possibilidade de ação. Refere-se à sensação de que o indivíduo está localizado em um ambiente espacial contíguo, onde se percebe o conteúdo como real (Freeman, Lessiter, Pugh, \& Keogh, 2005), ainda que não haja a sensação de ser transportado para outro mundo (Lee, 2004).

A presença social é compreendida como a percepção de estar com outra pessoa em um ambiente mediado. A experiência subjetiva de estar junto com outros em um ambiente virtual, mesmo quando os usuários estão fisicamente situados em lugares diferentes (Biocca et al., 2003) engloba três aspectos principais, a copresença, o envolvimento psicológico e o engajamento comportamental.

Copresença. Percepção sensorial da existência de outra pessoa. É importante que essa percepção possa ser compreendida como mútua. Ao mesmo tempo em que um individuo pode perceber a presença de outra pessoa esse outro pode perceber sua copresença (Biocca et al., 2003; Heeter, 1992).

Envolvimento psicológico. Envolvimento de funções cognitivas na interação com o outro. O sujeito tem a sensação de acessar a inteligência de outra pessoa. A saliência das relações interpessoais, a possibilidade de compreender e ser compreendido pelo outro e mostrar e perceber algum grau de intimidade (Biocca et al., 2003; Lee, 2004).

Engajamento comportamental. Principio de reação e interatividade. O sujeito propõe algo que pode ser percebido pelo outro e o outro pode ou não reagir e vice-versa. Possibilidade de ação sobre a relação com o outro (Biocca et al., 2003).

Em um experimento que verificou a relação entre a presença social e a percepção de proximidade, Gooch e Watts (2014) identificaram que o impacto instantâneo da presença social durante o ato de comunicação impacta em uma sensação duradoura de proximidade em trocas de mensagens instantâneas. O estudo sugere que diferentes tipos de mídia podem afetar essa relação.

Já a autopresença é o estado psicológico no qual o self virtual é experienciado como self real. Refere-se à representação mental de si mesmo no mundo virtual, no que diz respeito à percepção de seu corpo (proto-self), estados psicológicos e 
emocionais (core self), percebidos como traços e identidade (extended self). Existe autopresença quando o individuo percebe sua representação na tecnologia como do seu corpo, de suas emoções e sua identidade (Ratan, 2012; Lee, 2004). Em um levantamento dos instrumentos que vêm sendo utilizados para mensurar presença social e espacial, van Baren e ljsselsteijn (2004) indicam uma série de indices objetivos e subjetivos. As medidas subjetivas descritas são autorrelatos avaliados por meio de questionários, medidas qualitativas, contínuas e psicofísicas. Já as objetivas, consistem em respostas produzidas pelos indivíduos que não passam por deliberação consciente, como correlatos neurais, medidas comportamentais e de desenvolvimento de tarefas.

Em uma revisão sobre artigos empíricos publicados sobre presença, Schütz (2018) encontrou a utilização de medidas qualitativas como entrevista em profundidade (Felton, 2014), grupos focais (Best, Taylor, \& Manktelow, 2015), reconhecimento de imagens cognitivas (Shin, Lee, \& Kim, 2015) e percepção em ambiente simulado (Han, Shih, Rosson, \& Carroll, 2014). Dois artigos mencionam escalas de autorrelato. Lee e Shin (2014) desenvolveram uma escala, com quatro itens sobre presença social, que varia de 1 (discordo fortemente) a 7 (concordo fortemente) e avalia a percepção de estar lá com políticos em suas postagens no Twitter. Kim, Suh e Lee (2013) desenvolveram um instrumento com seis itens relativos à copresença experimentada em um ambiente virtual.

Outros instrumentos têm sido considerados indicativos de mensuração de acordo com o tipo de presença avaliado. Para a mensuração de presença social, destaca-se a Semantic Differential Technique de Short et al. (1976) (replicada ou adaptada por Kim, Frank, \& Kim, 2014; Ogara et al., 2014) e Cyr, Head e Ivanov (2007) (replicada por Toufaily et al., 2013). Já para a mensuração de presença física ou espacial, a Presence Questionnaire (PQ) de Witmer e Singer (1998) tem sido utilizada na literatura recente (Stravopoulos et al., 2013) e apresentouse, originalmente, como um instrumento com bons índices de consistência interna. Apesar da utilização de diversas medidas para a avaliação de presença, somente encontrou-se a tradução da $P Q$ para o idioma português, em contexto de realidade virtual (Silva et al., 2016) e não foram encontradas adaptações para tecnologias ubíquas. Além do tipo de presença, características do meio (Benyon, 2012; Han, Min, \& Lee, 2016; Park \& Sundar, 2015) e dos usuários (Lowden \& Hostetter, 2012; Nowak, 2013; Ratan, 2012) têm sido levadas em conta nos estudos sobre presença.

A ubiquidade de um dispositivo depende de algumas características básicas que perpassam a percepção do usuário sobre o seu uso, além de características das suas possibilidades de usabilidade. Para tanto, é necessário que sejam capazes de fornecer continuidade e simultaneidade, imediatez e velocidade, portabilidade e mobilidade e, ainda, busca e acessibilidade (Okazaki \& Mendez, 2013). Portanto, os artefatos com características de ubiquidade mais disseminados são smartphones, tablets e wearables, cuja imersão no cotidiano é tão indiscriminada de forma que a diferenciação entre usuário e ferramenta, por vezes se torna sutil (Rutledge, 2013).

O estudo sobre tecnologias ubiquas se justifica quando se considera sua disseminação - 96\% dos usuários acessam à internet por meio de smartphones (CGI.br, 2019) - e suas possibilidades como meio de divulgação de mensagens sobre bem-estar e mudanças de estilos de vida (CGI.br, 2018). As particularidades do mundo online têm o potencial de influenciar capacidades atencionais, o processamento da memória e a cognição social, uma vez que a internet é um meio de interação que se assemelha e evoca processos do mundo off-line, criando uma interrelação com as vidas sociais e influenciando autoconceito e autoestima (Firth et al., 2019). O conceito de presença pode ser considerado um dos principais componentes que intermedeiam a relação entre os espaços virtuais e as pessoas (Freeman et al., 2005; Lee, 2004; Schütz, 2018), especialmente quando se considera a relação com tecnologias ubiquas.

Dessa forma, o objetivo desse estudo foi desenvolver uma escala de presença em tecnologias ubíquas e verificar as propriedades 
psicométricas do instrumento por meio de análise de evidências de validade e de fidedignidade. Especificamente, buscou-se analisar de forma exploratória e confirmatória a estrutura fatorial do instrumento nessa amostra, bem como sua consistência interna e adequação com o modelo proposto inicialmente.

\section{Método}

Este estudo contou com a realização de duas etapas metodológicas. Em uma primeira etapa, descrevem-se os procedimentos relativos à elaboração e à construção do instrumento proposto. Na segunda etapa, apresentam-se as evidências de validade da escala, tendo por referência sua estrutura interna. Para verificar a validade da escala utilizou-se uma abordagem de visão unificada, na qual se compreende que o acúmulo de diversas evidências de validade reforça a validade do construto e embasa as possiveis explanações e conclusões sobre os escores obtidos pela escala (International Test Commission, 2017). Assim, compreende-se que todas as evidências de validade buscam a validação do construto, ou seja, quanto mais evidências de validade, mais provável de se constatar que a escala mede aquilo que se propõe e que os escores obtidos pela escala não acontecem ao acaso.

$1^{a}$ etapa: construção da escala. $\bigcirc$ instrumento foi elaborado de acordo com as recomendações apontadas pela International Test Commission (ITC, 2005, 2017) e Pasquali (2010). Inicialmente, foi realizada a escolha dos atributos comportamentais referentes ao objeto de estudo e à operacionalização do construto. Para especificar os possiveis fatores associados à presença e criar os itens da escala realizou-se uma revisão da literatura. Em seguida, para avaliar possiveis sobreposições na definição do conteúdo do construto e a adequação dos itens à população de interesse, a escala foi apresentada a três juizes, dois grupos focais e a uma aplicação-piloto em uma amostra reduzida. Esses procedimentos serão apresentados a seguir.

Elaboração da versão preliminar do instrumento. $O$ instrumento em questão foi denominado Escala de Presença em Tecnologias Ubiquas (EPTU). Sua versão preliminar foi inspirada em alguns aspectos de instrumentos prévios existentes na literatura, tais como o formato das escalas, as definições operacionais aplicadas nos itens e a utilização ou não de tecnologias. Entretanto, a elaboração dos itens não se baseou em instrumentos prévios especíicos.O instrumento conta com 30 itens que foram respondidos em uma escala Likert de 11 pontos (variando de 0 = Discordo Totalmente a 10 = Concordo Totalmente). Os itens foram divididos em três dimensões: presença espacial, presença social e autopresença. Como o objetivo do estudo envolve a avaliação da presença nas tecnologias ubiquas, o enunciado do instrumento direciona para o uso desse tipo de tecnologia: ao usar o meu celular eu percebo que.

Cada uma das dimensões foicomposta por 10 itens. A dimensão de presença espacial propõese a verificar percepções com relação a situar-se em outro ambiente (ex., parece que estou em outro ambiente). A presença social com relação à percepção de copresença (ex., alguém está comigo), envolvimento psicológico (ex., alguém pode me fazer companhia,) e engajamento comportamental (ex., posso chamar alguém quando quiser). A autopresença propunha-se a mensurar a percepção da representação na tecnologia como reflexo do corpo, emoções e identidade (ex., posso me expressar).

Avaliação de juízes. Realizou-se a verificação da validade de conteúdo, conforme proposto por Pasquali (2010), por meio de três juizes, um especialista na área de comunicação e tecnologia e dois psicólogos com experiência em avaliação psicológica. Como rapport do questionário os juizes receberam uma definição ampla dos construtos avaliados e o instrumento original. Para cada um dos itens, os juizes deveriam atribuir uma pontuação de 1 (desnecessário), 2 (útil, mas não essencial) ou 3 (essencial) para avaliar a pertinência do item com relação ao construto. Os itens para os quais algum dos juizes atribuiu a pontuação 1 (desnecessário) foram 
excluídos. Como critério de inclusão, utilizou-se a concordância de pelo menos dois dos juizes com o item de pontuação 3, essencial. Ao total foram excluidos quatro itens nessa etapa (posso produzir conteúdo, posso ficar sabendo do que acontece com alguém, meus dedos são como parte do conteúdo, é difícil me dar conta do que acontece em minha volta).

Análise Semântica. Os 26 itens remanescentes foram apresentados a dois grupos focais para verificar a compreensão dos itens. O primeiro grupo focal contou com 8 participantes, de ambos os sexos, de 24 a 35 anos. O segundo grupo focal contou com 6 participantes, de ambos os sexos, com idades entre 19 e 32 anos. Os participantes de ambos os grupos focais asseguraram sua participação por meio do Termo de Consentimento Livre e Esclarecido e sugeriram alterações na apresentação de alguns termos, considerando a exclusão da palavra dados dos itens e o formato de apresentação e de ordenação das palavras de um dos itens. Todas as alterações sugeridas pelos grupos focais foram atendidas e, portanto, 5 itens sofreram alterações. Além disso, os grupos apontaram dificuldade de compreensão em outros 6 itens, que foram retirados (três itens de presença espacial: existem coisas que posso fazer tanto nesse ambiente virtual quanto em outros ambientes; minha atenção fica totalmente voltada aos conteúdos que estou acessando; e estou fisicamente em outro mundo; dois itens de autopresença: os dados que acesso estão fisicamente próximos a mim; posso tocar no conteúdo; e um item de presença social: parece que alguém está fisicamente próximo a mim).

Etapa piloto. A etapa seguinte consistiu na aplicação piloto, em 30 participantes, com idade média de 26 anos $(D P=4,61)$, sendo que
73.3\% se identificaram com o gênero feminino. Quanto à escolaridade, 6,7\% possuía Ensino Médio Completo, 33.3\% Ensino Superior Incompleto, 13.3\% Ensino Superior Completo e 46,7\% possuiam Pós-graduação. A amostra foi selecionada por conveniência, por meio de anúncio em uma página de rede social digital. Análises iniciais de correlação entre os itens averiguaram possiveis multicolinearidades e verificaram a consistência interna da escala. Verificou-se que cinco itens apresentavam correlações superiores a 0,9 entre si. Por considerar que itens com correlações altas e significativas se propõem a mensurar os mesmos aspectos, optou-se pela retirada de dois itens (alguém pode me fazer sentir bem; posso demonstrar tristeza por meio dos conteúdos), resultando em um total de 18 itens. Após a exclusão de todos os itens mencionados, a escala apresentou alta consistência interna $(\alpha=0,94)$.

$2^{a}$ etapa: evidências de validade. A versão final do instrumento se propõe a mensurar a presença percebida na interação com tecnologias ubiquas. O instrumento considera 3 construtos subjacentes ao construto principal de presença: presença social (verificado por meio de 7 itens), presença espacial (6 itens) e autopresença (5 itens). A aplicação do instrumento é individual. O modelo sugere a mensuração de quatro variáveis latentes, sendo 3 variáveis latentes, de primeira ordem.

\section{Participantes}

A amostra foi constituída por 458 participantes, com idade média de 25,2 anos $(D P=4,87)$, dos quais $74,7 \%$ identificam-se com o gênero feminino, e $84,6 \%$ eram do estado do Rio Grande do Sul. Os dados demográficos dos participantes são apresentados na Tabela 1. 
Tabela 1 - Distribuição dos participantes por escolaridade, renda e região

\begin{tabular}{lll}
\hline & & \\
\hline Escolariável & Ensino Médio Incompleto & $\%$ \\
& Ensino Médio Completo & 9,70 \\
& Ensino Superior Incompleto & 19,7 \\
& Ensino Superior Completo & 43,4 \\
\hline Renda & Pós Graduação & 27,3 \\
\hline Região & Até 2 salários mínimos & 14,8 \\
& De 2 a 4 salários mínimos & 27,7 \\
& De 4 a 10 salários mínimos & 38 \\
& De 10 a 20 salários minimos & 15,1 \\
& Mais de 20 salários mínimos & 4,4 \\
\hline & Sul & 89,1 \\
& Sudeste & 7,4 \\
& Nordeste & 2,3 \\
& Norte & 0,2 \\
\hline
\end{tabular}

\section{Procedimentos}

Os participantes foram convidados a responder um questionário online em uma página do Grupo de Pesquisa em Psicologia Comunitária da UFRGS em uma rede social e eram convidados a compartilhar a pesquisa com seus amigos. Para a hospedagem do instrumento online contou-se com o site typeform, uma plataforma que mediante a criação de uma conta, permite a criação de questionários de forma gratuita. A primeira página da pesquisa referia-se ao TCLE. A pesquisa foi aprovada pelo Comitê de Ética em Pesquisa do Instituto de Psicologia da Universidade Federal do Rio Grande do Sul (UFRGS), e está registrada na Plataforma Brasil sob o número 52303215.9.0000.5334. Aqueles que consentissem com a participação eram encaminhados à continuidade da pesquisa. Foram apresentadas questões sociodemográficas referentes à idade, sexo, estado no qual reside e questões referentes à utilização de tecnologias ubiquas. Com relação ao dispositivo de resposta, 53,3\% dos participantes respondeu à pesquisa por meio de smartphone,
$44.5 \%$ por meio de computadores e laptops e 2,2\% por meio de tablets.

Inicialmente, foram realizadas análises de consistência interna por meio do alfa de Cronbach, considerando as dimensões com todos os itens e também com os itens excluídos. Em seguida, conduziram-se Análises Fatoriais Confirmatórias (AFC) para verificar o comportamento de variáveis observadas e latentes a partir de modelos estabelecidos a priori pela literatura. O procedimento considerou na análise erros de ajuste dos modelos e verificou variáveis observadas e variáveis latentes (Byrne, 2010). $O$ ajuste do modelo foi verificado por meio do Índice de Comparação do Ajuste (CFI, com valor esperado acima de 0,95) e a análise dos resíduos por meio do Erro Quadrático Médio de Aproximação e Raiz Quadrada Média Padronizada (RMSEA e SRMR, com valor esperado inferior a 0,08 , considerando intervalo de confiança de 90\%) (Byrne, 2010). Também são apresentados os valores do qui-quadrado ( $\chi 2$ ), graus de liberdade e sua respectiva significância estatística. Para 
tanto, utilizou-se o programa $\mathrm{R}$ versão 3.4.3 para analisar os dados por meio do método de estimação de mínimos quadrados ponderados (Weighted Least Squares Means and Varianceadjusted - WLSMV). A escolha pelo método ocorreu considerando ser o mais apropriado para respostas de distribuição normais e não normais, com tamanhos de amostra entre 100 e 1.000 participantes (Byrne, 2010). Considerouse ainda que quando a validade do construto é observada por dados ordinais, como nas escalas Likert, a utilização de correlações policóricas em detrimento de correlações de Pearson apresenta um melhor ajuste ao modelo teórico e reproduz melhor o modelo de mensuração dos dados, independentemente do número de fatores (Holgado-Tello, Chacón-Moscoso, BarberoGarcia, \& Vila-Abad, 2010).

Também foram realizadas Análises Fatoriais Confirmatórias Multigrupo para verificar a invariância fatorial das escalas, considerandose gênero. A partir dos dados obtidos com os resultados das AFC das escalas, a Análise Fatorial Confirmatória Multigrupo (AFCMG) avaliou se os parâmetros de cada escala são equivalentes de acordo com o gênero (feminino e masculino). Para verificar a equivalência de parâmetros entre grupos, foram testados três modelos para cada escala, sendo que cada modelo é mais restritivo do que o outro. No primeiro modelo, os parâmetros são livremente estimados para cada grupo, sendo testada apenas a plausibilidade da estrutura fatorial, ou seja, a adequação do modelo proposto para cada grupo. No segundo modelo, as cargas fatoriais são restringidas para serem iguais entre os grupos, avaliando se os itens possuem a mesma importância para os grupos. No último modelo, além das cargas fatoriais, também são restringidos os interceptos, ou seja, as médias dos itens para cada grupo. Para este estudo, foram levados em conta os indices comparativos de ajuste entre os modelos, considerando-se a diferença entre o CFI de um modelo para o outro. A diferença entre o CFI
( $\triangle$ CFI) não deve ser maior de 0,01 , e os resultados globais indicam a viabilidade de se restringir as cargas fatoriais para serem as mesmas nos diferentes grupos (Milfont \& Fisher, 2010).

\section{Resultados}

Análise de consistência interna. A análise de consistência interna foi realizada por meio do alfa de Cronbach. Observa-se que o alfa de Cronbach do fator autopresença é de 0,849. O alfa do fator presença social é de 0,844. Para o fator presença espacial, o alfa é de 0,756, aumentando para 0,759 com a exclusão dos itens "PE3" e "PE6". O alfa total da escala foi de 0,905

Análise Fatorial Confirmatória (AFC). A literatura sugere que presença pode ser um construto de primeira ou de segunda ordem (Lee, 2004). Portanto, a AFC testou tanto um modelo de primeira ordem, com correlação entre os três fatores latentes da Escala de presença, quanto um modelo de segunda ordem. Os resultados para o modelo inicial, com os 18 itens, apresentaram índices iniciais aceitáveis, como pode ser observado na Tabela 2, já que os indices de ajuste ficaram acima 0,95 e os residuos ficaram abaixo de 0,08, incluindo seus limites superiores.

Ao considerar que a exclusão dos itens $P E 3$ e PE6 resulta em uma melhora da consistência interna do fator presença espacial, optou-se por excluir os itens na AFC. Ainda, os indices de modificação indicam que os itens de presença social PS1 e PS5 carregam no fator autopresença com carga maior do que no seu fator original teórico, o que também sugeriu a exclusão desses itens. Levando em conta esses critérios, os quatro itens foram excluídos do modelo, obtendo-se os ajustes apresentados no modelo com 14 itens (Anexo I). Dessa forma, testou-se o Modelo Final com 14 itens, que apresentou indices de ajuste satisfatórios (Tabela 2) com todos os pesos padronizados dos itens sendo significativos e acima de 0,40 tanto para o modelo de $1 .{ }^{a}$ ordem, quanto para o modelo de $2 .^{\mathrm{a}}$ ordem. 
Tabela 2 - Análises Fatoriais Confirmatórias - Índices de ajuste para Escala de Presença

\begin{tabular}{|c|c|c|c|c|c|c|}
\hline Modelo & $x^{2}$ & gl & $\boldsymbol{p}$ & CFI & RMSEA $\left(I . C .^{a}\right)$ & SRMR \\
\hline $\begin{array}{l}\text { Escala Presença } 1^{\mathrm{a}} \text { ordem - } \\
\text { Modelo inicial } 18 \text { itens }\end{array}$ & 297,38 & 132 & $<0,01$ & 0,976 & $0,052(0,044-0,059)$ & 0,069 \\
\hline $\begin{array}{l}\text { Escala Presença } 1^{\text {a }} \text { ordem - } \\
\text { Modelo Final } 14 \text { itens }\end{array}$ & 116,83 & 74 & $<0,01$ & 0,990 & $0,035(0,022-0,047)$ & 0,053 \\
\hline $\begin{array}{l}\text { Escala Presença } 2^{\mathrm{a}} \text { ordem - } \\
\text { Modelo inicial } 18 \text { itens }\end{array}$ & 297,39 & 132 & $<0,01$ & 0,975 & $0,053(0,044-0,060)$ & 0,069 \\
\hline $\begin{array}{l}\text { Escala Presença } 2^{a} \text { ordem - } \\
\text { Modelo Final } 14 \text { itens }\end{array}$ & 116,84 & 74 & $<0,01$ & 0,990 & $0,036(0,022-0,048)$ & 0,054 \\
\hline
\end{tabular}

Na Figura 1, é apresentado o gráfico do modelo

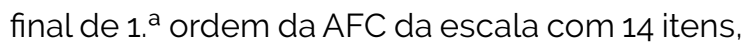
com os parâmetros padronizados. Os itens estão apresentados por números que correspondem à Tabela 3. Os pesos padronizados variaram entre 0,575 para o item PS2 a 0,870 para o item AP3.
Além disso, observam-se correlações positivas e significativas entre as três variáveis latentes, indo de 0.526 entre os fatores autopresença e presença espacial, a 0,743 entre os fatores autopresença e presença social.

Figura 1 - AFC do modelo final de $1 .^{\text {a }}$ ordem dos três fatores da Escala de Presença com 14-itens com cargas padronizadas.

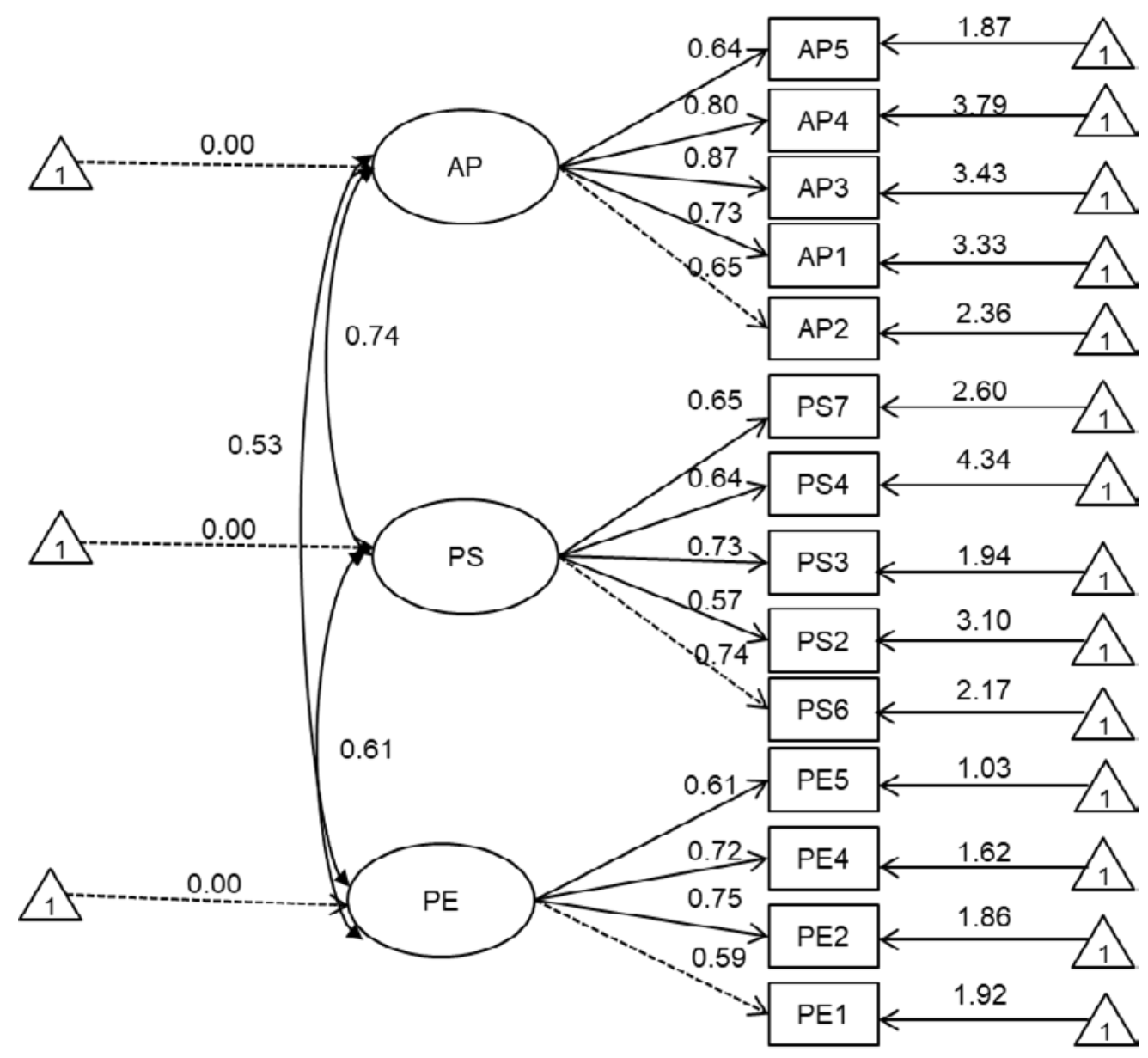


Os parâmetros padronizados estimados de cada item para a AFC da Escala são apresentados para a amostra total e para homens e mulheres na Tabela 3. Todos os parâmetros são significativos. Nessa tabela, podem-se observar as cargas fatoriais de cada item em seu respectivo fator latente, conforme apresentado na Figura 1. Os itens estão apresentados por números que correspondem à Tabela 3

Tabela 3 - Parâmetros padronizados, modelo de $1^{\text {a }}$ ordem com 18 itens

\begin{tabular}{|c|c|c|c|c|c|}
\hline \multirow{2}{*}{ Itens } & \multirow{2}{*}{\multicolumn{2}{|c|}{ Fator }} & \multicolumn{3}{|c|}{ Pesos Padronizados } \\
\hline & & & Total & Mulheres & Homens \\
\hline $\begin{array}{l}\text { AP1 - Posso demonstrar alegria por meio dos } \\
\text { conteúdos }\end{array}$ & $<---$ & & 0,732 & 0,870 & 0,684 \\
\hline $\begin{array}{l}\text { AP2 - Posso demonstrar raiva por meio dos } \\
\text { conteúdos }\end{array}$ & $<---$ & & 0,651 & 0,601 & 0,667 \\
\hline $\begin{array}{l}\text { AP3 - Posso me expressar por meio dos } \\
\text { conteúdos }\end{array}$ & $<---$ & Autopresença & 0,870 & 0,937 & 0,843 \\
\hline $\begin{array}{l}\text { AP4 - Posso demonstrar minhas opiniões por } \\
\text { meio dos conteúdos }\end{array}$ & $<---$ & & 0,804 & 0,901 & 0,763 \\
\hline AP5 - Os conteúdos são parte de mim & $<---$ & & 0,644 & 0,545 & 0,685 \\
\hline $\begin{array}{l}\text { PS2 - Pessoas importantes estão próximas a } \\
\text { mim }\end{array}$ & $<---$ & & 0,575 & 0,606 & 0,567 \\
\hline PS3 - Não estou sozinho & $<---$ & & 0,729 & 0,671 & 0,749 \\
\hline $\begin{array}{l}\text { PS4 - Alguém pode conversar comigo e } \\
\text { posso conversar com alguém }\end{array}$ & $<---$ & Presença Social & 0,640 & 0,766 & 0,594 \\
\hline PS6 - Alguém pode me fazer companhia & $<---$ & & 0,742 & 0,755 & 0,740 \\
\hline PS7 - Posso chamar alguém quando quiser & $<---$ & & 0,653 & 0,580 & 0,680 \\
\hline $\begin{array}{l}\text { PE1 - Estou conectado com um local diferen- } \\
\text { te de onde estou }\end{array}$ & $<---$ & & 0,590 & 0,624 & 0,588 \\
\hline PE2 - Posso modificar esse ambiente virtual & $<---$ & Presença Espacial & 0,748 & 0,645 & 0,777 \\
\hline PE4 - Parece que estou em outro ambiente & $<---$ & & 0,724 & 0,731 & 0,733 \\
\hline $\begin{array}{l}\text { PE5 - Posso fazer o que eu quiser nesse } \\
\text { ambiente virtual }\end{array}$ & $<---$ & & 0,609 & 0,685 & 0,601 \\
\hline
\end{tabular}

\section{Análise Fatorial Confirmatória Multigrupo} (AFCMG). A partir dos dados obtidos com os resultados da AFC da escala, a análise fatorial confirmatória multigrupo (AFCMG) foi realizada com o objetivo de avaliar se os parâmetros psicométricos da escala são equivalentes para diferentes grupos. Foram testados os grupos considerando o gênero para o modelo final com 14 itens tanto de $1 .{ }^{\text {a }}$ ordem como de $2 .^{\text {a }}$ ordem. A partir da Tabela 4, percebe-se que a escala apresenta índices adequados de ajuste para o modelo de $1 .^{\text {a }}$ ordem não restringido ao ser considerado o gênero. Quando as cargas fatoriais são restringidas para serem iguais entre os grupos, não há diferença entre o $C F I(\triangle C F I<0,01)$, permanecendo um ajuste aceitável. Da mesma forma, quando da restrição dos interceptos, não há diferença entre os homens e as mulheres, atingindo, dessa forma, um ajuste adequado para o modelo de $1 .^{a}$ ordem. Já para o modelo 
de 2. ${ }^{a}$ ordem, os resultados apontam que há equivalência para o modelo escalar com cargas fatoriais e interceptos restringidos, o que indica que é possivel comparar as médias dos fatores latentes para gênero. Todos os parâmetros foram significativos nos modelos multigrupo.

Tabela 4 - Análises Fatoriais Confirmatórias Multigrupo - Índices de ajuste para gênero

\begin{tabular}{|c|c|c|c|c|c|c|}
\hline Modelo & $x^{2}$ & $g l$ & $p$ & CFI & RMSEA (I.C. $\left.{ }^{\text {a }}\right)$ & SRMR \\
\hline $\begin{array}{l}\text { Escala Presença } 1^{a} \text { ordem - modelo } \\
\text { não restringido }\end{array}$ & 145.38 & 148 & 0,54 & 0,999 & $0,001(0,001-0,029)$ & 0,058 \\
\hline $\begin{array}{l}\text { Escala Presença } 1^{\text {a }} \text { ordem - cargas } \\
\text { fatoriais restringidas }\end{array}$ & 184,35 & 159 & 0,08 & 0,994 & $0,026(0,001-0,041)$ & 0,065 \\
\hline $\begin{array}{l}\text { Escala Presença } 1^{\mathrm{a}} \text { ordem - cargas } \\
\text { fatoriais e interceptos restringidos }\end{array}$ & 203,62 & 170 & 0,04 & 0,992 & $0,029(0,007-0,043)$ & 0,067 \\
\hline $\begin{array}{l}\text { Escala Presença } 2^{a} \text { ordem - modelo } \\
\text { não restringido }\end{array}$ & 145,38 & 148 & 0,54 & 0,999 & $0,001(0,001-0,029)$ & 0,058 \\
\hline $\begin{array}{l}\text { Escala Presença } 2^{a} \text { ordem - cargas } \\
\text { fatoriais restringidas }\end{array}$ & 203.75 & 161 & 0,01 & 0,990 & $0,034(0,016-0,047)$ & 0,068 \\
\hline $\begin{array}{l}\text { Escala Presença } 2^{a} \text { ordem - cargas } \\
\text { fatoriais e interceptos restringidos }\end{array}$ & 223,62 & 171 & 0,01 & 0,988 & $0,036(0,021-0,049)$ & 0,070 \\
\hline
\end{tabular}

a I.C. = Intervalo de Confiança de $90 \%$

\section{Discussão}

Este estudo desenvolveu uma escala de presença em tecnologias ubiquas e verificou suas propriedades psicométricas por meio da análise de evidências de validade e fidedignidade. Os dados apontam que o modelo da escala com 14 itens e três fatores de presença independentes apresenta indices de ajuste adequados e bons índices de consistência interna para a amostra observada. Esses resultados apresentam um avanço no estudo da área, especialmente com relação a possiveis influências das tecnologias ubiquas. Ainda que se compreenda que a presença se relacione mais com capacidades cognitivas do que com características do meio (Riva et al., 2019), os resultados desse estudo propõem a mensuração do fenômeno associado a questões da ubiquidade, que parecem influenciar na percepção dos participantes com relação à presença.

O modelo final com as três variáveis latentes observadas, autopresença, presença espacial e presença social, apresenta indices de ajuste satisfatórios, com cargas fatoriais acima de 0,5 e as três variáveis latentes correlacionam-se positiva e significativamente, com a maior correlação entre a presença social e a autopresença. Pode-se argumentar que a tendência de simular atores sociais virtuais e até mesmo autorrepresentações seja mais forte do que a de simular objetos físicos não humanos que poderiam configurar a percepção espacial, porque a consciência de outros seres humanos tem maior importância para a sobrevivência humana, afinal, os seres humanos são seres sociais (Lee, 2004). A percepção de presença por meio de tecnologias ubíquas pode favorecer menos a percepção de presença espacial do que outros dispositivos de realidade virtual, que apresentam estímulos de objetos diferenciados e propiciam a utilização de praticamente todos os sentidos, facilitando a ideia de localizar-se em outro espaço, uma vez que o ambiente virtual simulado apresenta estímulos mais ricos (Riva et al., 2019).

Dentre os itens que avaliam a presença social, aquele que teve a maior carga fatorial foi: alguém pode me fazer companhia $(\lambda=0,742)$. $O$ item remete à percepção sensorial da existência de outra pessoa. Biocca et al. (2003) descrevem 
essa percepção sensorial como uma forma de avaliação de disponibilidade mútua. Dessa forma, é possivel conceber que a ubiquidade das tecnologias utilizadas como base para o desenvolvimento dos itens dessa escala possa influenciar em suas respostas, uma vez que uma importante caracteristica da ubiquidade seja a continuidade do acesso, o tempo de resposta e, portanto, o quanto as mesmas ocorrem de forma simultânea e imediata (Okazaki \& Mendez, 2013), aproximando-se das interações que ocorrem sem mediação das tecnologias. Além disso, a percepção de presença social com mensagens instantâneas parece contribuir para a percepção de mais proximidade (Gooch \& Watts, 2014).

Para Riva et al. (2019) os ambientes fornecem oportunidades variadas de presença. Assim, as habilidades do usuário e suas intenções podem ser facilitadas pelas diferentes características dos ambientes. Dessa forma, as tecnologias ubiquas fornecem aos usuários possibilidades de interações constantes que podem estar associadas a percepções mais acuradas para a disponibilidade do outro, facilitando a presença social. A alta sincronia tem sido apontada na relação entre prestadores de serviço e consumidores, como associada à copresença. A utilização de emoticons e mensagens de texto facilitam a ideia de reciprocidade e troca de estados afetivos entre usuários e prestadores de serviço (Park \& Sundar, 2015).

O item que apresentou maior carga fatorial para a presença espacial foi: posso modificar esse ambiente virtual $(\lambda=0,748)$. A possibilidade de ação em um determinado espaço é uma das características essenciais para a presença espacial (Freeman et al., 2005; Hartman et al., 2015) e parece ser um indicativo associado à ubiquidade, de acordo com os resultados dessa escala. Ainda que a maioria dos usuários de tecnologias ubíquas não esteja familiarizada com o desenvolvimento de softwares e hardwares, as possibilidades de personalização do aparelho e dos aplicativos podem influenciar na percepção de atuação sobre o espaço. A experiência de presença não depende tanto da percepção e da crença na reprodução fiel de aspectos físicos ou objetos da realidade externa, mas na capacidade de simulação de produção de um contexto no qual os atores sociais possam se comunicar e cooperar (ljsselsteijn \& Riva, 2003; Riva et al., 2019). As qualidades dinâmicas e hibridas de espaços virtuais que misturam o experimentado na relação mediada e no ambiente não mediado podem contribuir para a sensação de se sentir próximo de pessoas que estão distantes ao mesmo tempo em que se sente conectado com o local físico que se ocupa (Benyon, 2012).

Para os itens que avaliam a autopresença, o item com maior carga fatorial foi: posso me expressar por meio dos conteúdos $(\lambda=0,870$ ). A possibilidade de se expressar é um dos fatores importantes para a autopresença, por estar relacionado tanto a representações da sua autopercepção, seus estados emocionais e, ainda, traços da identidade, fatores essenciais para a percepção da autopresença (Ratan, 2012; Lee \& Shin, 2014). A mensuração da autopresença na EPTU leva em conta o quanto a possibilidade de ação e de expressão pode estar associada com a autopresença.

Existem informações que podem ser expressas e compartilhadas de acordo com a forma como se compreende o local onde se está inserido. Outros estudos já apontaram as associações entre niveis individuais de presença social com o estilo de uma atividade ou jogo escolhido e com as possibilidades de customização de um avatar, por exemplo (Ratan, 2012). O presente estudo corrobora esse achado e reforça a hipótese da importância contextual, considerando que os fatores presença social e autopresença apresentam altas correlações entre si.

Os resultados da AFCMG apresentam que os parâmetros psicométricos da escala são equivalentes entre homens e mulheres, o que sugere que, apesar da amostra ser composta por um número maior de mulheres, esse resultado não influencia na estabilidade da medida. Com relação a diferenças entre gêneros na percepção de presença, a literatura não apresenta consenso, sendo que alguns estudos indicam diferenças significativas entre homens e mulheres, com médias superiores tanto para homens (Nowak, 2013) quanto 
para mulheres (Lowden \& Hostetter, 2012).

Os resultados desse estudo reforçam a concepção de que a presença é um construto multifatorial, cujos fatores podem ser compreendidos de forma independente. Além disso, os resultados aqui apresentados aplicam-se para a mensuração da presença com relação à utilização de tecnologias ubíquas e, ainda que as características do meio não sejam determinantes para a existência do fenômeno, elas podem influenciar sua manifestação, uma vez que a presença é um fenômeno contextual. Cada ambiente fornece informações específicas para cada indivíduo, com oportunidades diferentes de ação e, considerando as diferenças individuais, também possibilidades variadas de intenção. Assim, a presença não pode ser separada da experiência do sujeito, mas é relacionada a ela (Riva, 2019).

\section{Considerações finais}

Esse estudo não pretende delimitar as discussões sobre a presença, tampouco fornecer uma solução definitiva para a sua mensuração. Entretanto, apresenta um instrumento em língua portuguesa, com propriedades psicométricas aceitáveis. Destaca-se os bons indices de ajuste do modelo e as consistências internas aceitáveis dos três fatores, autopresença, presença social e presença especial, apresentando-se como uma medida de autorrelato confiável.

Como limitações, os resultados podem ter sido influenciados pela diferença no tamanho da amostra, por gênero (74,3\% dos respondentes eram mulheres). Apesar da utilização de recrutamento online, os participantes acabaram sendo majoritariamente do Rio Grande do Sul (84,6\%), impedindo a generalização dos resultados para um contexto nacional.

No entanto, esse estudo apresenta um dos primeiros instrumentos a avaliar o fenômeno de presença em tecnologias ubiquas do Brasil, ainda que levando em conta essas ressalvas. A verificação das propriedades psicométricas da escala no contexto brasileiro é fundamental para que se possa aprofundar a discussão sobre o alcance das tecnologias ubíquas no cotidiano do País e do fenômeno da presença para o desenvolvimento de aplicações que visem a promoção de saúde e de bem-estar de acordo com as necessidades contextuais.

Estudos futuros podem verificar o funcionamento da escala por meio de sua aplicação com medidas repetidas, considerando os mesmos sujeitos em diferentes momentos do dia, semanas, ano, possibilitando a verificação da estabilidade da escala. Além disso, a utilização de estratégias de mensuração em tempo real tem sido adotada em alguns estudos com tecnologias ubíquas no sentido de incrementar a validade ecológica dos resultados, o que pode se apresentar como uma solução para vieses, como os de memória, por exemplo. Essa modalidade de aplicação permitiria, ainda, a utilização concomitante de instrumentos objetivos obtidos por meio das tecnologias utilizadas (tais como número de acessos, tempo dispendido online etc.), auxiliando a compreender a presença de forma mais abrangente.

\section{Referências}

Benyon, D. (2012). Presence in blended spaces. Interacting with Computers, 24(4), 219-226. http://doi. org/10.1016/j.intcom.2012.04.005

Best, P., Taylor, B., \& Manktelow, R. (2015). I've 500 friends, but who are my mates? Investigating the influence of online friend networks on adolescent wellbeing. Journal of Public Mental Health, 14(3), 135-148. http://doi.org/10.1108/JPMH-05-2014-0022

Biocca, F., Harms, C., \& Burgoon, J. (2003). Toward a more robust theory and measure of social presence: Review and suggested criteria Presence: Teleoperators and Virtual Environments, 12(5), 456-480. http://doi. org/10.1162/105474603322761270

Byrne, B. (2010). Structural Equation Modeling with AMOS: Basic Concepts, Applications, and Programming. New York: Taylor \& Francis Group. DOI INEXISTENTE

Cyr, D., Head, M., \& Ivanov, A., (2007). Perceived interactivity leading to e loyalty: Development of a model for cognitive-affective user responses. International Journal of Human-Computer Studies 67, 850-869. https://doi. org/10.1016/j.ijhcs.2009.07.004

Comitê Gestor da Internet no Brasil - CGI.br (2018). Medição Da Saúde Digital Recomendações metodológicas e estudos de caso. Martínez, A. L.; Ortiz, D. N., Senne, F., p.19. 
Comitê Gestor da Internet no Brasil - CGI.br (2019). TIC cultura 2018 = pesquisa sobre o uso das tecnologias de informação e comunicação nos equipamentos culturais brasileiros. São Paulo, p. 38.

Felton, E. (2014). A/Effective connections: Mobility, technology and well-being. Emotion, Space And Society, 13. 9-15. https://doi.org/10.1016/i.emospa.2014.09.001

Firth, J., Torous, J., Stubbs, B., Firth, J., Steiner, G., Smith, L., Alvarez-Jimenez, M., Gleeson, J., Vancampfort, D., Armitage, C. and Sarris, J. (2019). The "online brain": how the Internet may be changing our cognition. World Psychiatry, 18(2), 119-129.

Freeman, J., Lessiter, J., Pugh, K., \& Keogh, E. (2005). When presence and emotion are related, and when they are not. Presence 2005: The 8th Annual Workshop on Presence, London. DOI INEXISTENTE

Gooch, Daniel \& Watts, Leon. (2014). Social Presence and the void in distant relationships: How do people use communication technologies to turn absence into fondness of the heart, rather than drifting out of mind? Al \& Society. 29(1). 10.1007/s00146-013-0492-9.

Han, S., Min, J., \& Lee, H. (2016). Building relationships within corporate SNS accounts through social presence formation. International Journal of Information Management, 36(6), 945-962

Han, K., Shih, P., Beth Rosson, M., \& Carroll, J. (2014). Understanding Local Community Attachment, Engagement and Social Support Networks Mediated by Mobile Technology. Interacting With Computers, 28(3), 220-237. https://doi.org/10.1093/iwc/iwuo40

Hartman, T., Wirth, W. Vorderer, P., Klimmt, C., Schramm, H., \& Böcking, S. (2015). Spatial Presence Theory: State of the Art and Challenges Ahead. In M. Lombard, F. Biocca, J. Freeman, W. IJsselsteijn \& R. Schaevitz, Immersed in Media (1st ed.). Springer International Publishing. 10.1007/978-3-319-10190-3

Heeter, C. (1992). Being there: The subjective experience of presence. Presence: Teleoperators and Virtual Environments, 1, 262-271. https://doi.org/10.1162/ pres.1992.1.2.262

Holgado-Tello, F. P., Chacón-Moscoso, S., Barbero-Garcia, I., \& Vila-Abad, E. (2010). Polychoric versus Pearson correlations in exploratory and confirmatory factor analysis of ordinal variables. Quality \& Quantity, 44, 153-166. https://doi.org/10.1007/s11135-008-9190-y

Ijsselsteijn, W. A. \& Riva, G. (2003) Being There: The experience of presence in mediated environments. In: Riva, G., Davide, F., \& ljsselsteijn, W. A., (eds.), Being There - Concepts, Effects and Measurements of User Presence in Synthetic Environments. IOS Press, pp. 3-16. DOI INEXISTENTE

International Test Commission. (2017). The ITC Guidelines for Translating and Adapting Tests (Second edition). [www.InTestCom.org]. DOI INEXISTENTE

International Test Commission (2005). International Guidelines on Computer-Based and Internet Delivered Testing. [www.intestcom.org]. DOI INEXISTENTE
Kim, D., Frank, M., \& Kim, S. (2014). Emotional display behavior in different forms of Computer Mediated Communication. Computers in Human Behavior, 30 , 222-229. https://doi.org/10.1016/j.chb.2013.09.001

Kim, H., Suh, K., \& Lee, U. (2013). Effects of collaborative online shopping on shopping experience through social and relational perspectives. Information \& Management, 50(4), 169-180. https://doi.org/10.1016/j.im.2013.02.003

Lee, E., \& Shin, S. (2014). When the Medium Is the Message. Communication Research, 41(8), 1088-1110. https://doi.org/10.1016/j.im.2013.02.003.

Lee, K. M. (2004). Presence, Explicated. Communication Theory, 14(1): 27-50. https://doi.or$\mathrm{g} / 10.1111 / \mathrm{j} .1468-2885.2004 . \mathrm{tb00302.x}$

Lowden, R. \& Hostetter, C. (2012). Access, utility, imperfection: The impact of videoconferencing on perceptions of social presence. Computers In Human Behavior, 28(2), 377-383. https://doi.org/10.1016/j.chb.2011.10.007

Lombard, M., \& Jones, M. T. (2015). Defining presence. In M. Lombard, F. Biocca, W.A. ljsselsteijn, J. Freeman, \& R. Schaevitz (Editors), Immersed in Media: Telepresence Theory, Measurement and Technology. London: Springer

Milfont, T. \& Fischer, R. (2010). Testing invariance across groups: Applications in cross-cultural research. International Journal of Psychological Research, 3. 111-130. https://doi.org/10.21500/20112084.857

Nowak, K. (2013). Choosing Buddy Icons that look like me or represent my personality: Using Buddy Icons for social presence. Computers in Human Behavior, 29, 1456-1464. https://doi.org/10.1016/j.chb.2013.01.027

Ogara S., Koh C, Prybutok, V. R. (2014). Investigating factors affecting social presence and user satisfaction with Mobile Instant Messaging. Computers in $\mathrm{Hu}$ man Behavior, 36. 453-459. https://doi.org/10.1016/j. chb.2014.03.064

Okazaki, S. \& Mendez, F. (2013). Perceived Ubiquity in Mobile Services. Journal of Interactive Marketing, 27(2), 98-111. https://doi.org/10.1016/j.intmar.2012.10.001

Park, E., \& Sundar, S. (2015). Can synchronicity and visual modality enhance social presence in mobile messaging? Computers In Human Behavior, 45, 121-128. http://dx.doi.org/10.1016/j.chb.2014.12.001

Pasquali, L. (2010). Instrumentação Psicológica: Fundamentos e Práticas. Porto Alegre: Artmed. DOI INEXISTENTE

Ratan, R. (2012). Self-presence, explicated: Body, emotion, and identity extension into the virtual self. In Handbook of Research on Technoself: Identity in a Technological Society (pp. 321-335). IGI Global. https:// doi.org/10.4018/978-1-4666-2211-1.ch018

Riva, G., Wiederhold, B., \& Mantovani, F. (2019). Neuroscience of Virtual Reality: From Virtual Exposure to Embodied Medicine. Cyberpsychology, Behavior, And Social Networking, 22(1), 82-96. 10.1089/cyber.2017.29099.gri

Rutledge, P. (2013). The Psychology of Mobile Media. In: P. Bruck \& M. Rao (Eds.), Global Mobile: Current Transformations and Futures of Value. Information Today, Inc. DOI INEXISTENTE 
Schütz, F. F. (2018). Presença nas tecnologias ubiquas e suas relações com medo de ficar de fora, autoeficácia, apoio social e bem-estar (Tese de Doutorado). Universidade Federal do Rio Grande do Sul. Recuperado a partir de: http://hdl.handle.net/10183/186088

Shin, Y., Lee, B., \& Kim, J. (2015). Prosocial Activists in SNS: The Impact of Isomorphism and Social Presence on Prosocial Behaviors. International Journal Of Human-Computer Interaction, 31(12), 939-958.

Short, J., Williams, E., \& Christie, B. (1976). The social psychology of telecommunications. London: John Wiley \& Sons. DOI INEXISTENTE

Silva, G., Donat, J., Rigoli, M., de Oliveira, F., \& Kristensen, C. (2016). A questionnaire for measuring presence in virtual environments: factor analysis of the presence questionnaire and adaptation into Brazilian Portuguese. Virtual Reality, 20(4), 237-242. http://dx.doi. org/10.1007/s10055-016-0295-7

Stavropoulos, V., Alexandraki, K., \& Motti-Stefanidi, F. (2013). Flow and Telepresence contributing to Internet Abuse: Differences according to Gender and Age. Computers in Human Behavior, 29(5), 1941-1948. http:// dx.doi.org/10.1016/j.chb.2013.03.011

Toufaily, E., Ricard, L., \& Perrien, J. (2013). Customer loyalty to a commercial website: Descriptive meta-analysis of the empirical literature and proposal of an integrative model Journal of Business Research, 66 . 1436-1447. https://doi.org/10.1016/j.jbusres.2012.05.011

van Baren, J. and ljsselsteijn, W. (2004). OmniPres Guide and Compendium. [online] International Society for Presence Research. Available at: https://ispr.info/ about-presence-2/tools-to-measure-presence/omnipres-guide/ [Accessed 6 Aug. 2018]. DOI INEXISTENTE

Witmer, B. G., \& Singer, M. J. (1998). Measuring presence in virtual environments: A presence questionnaire. Presence: Teleoperators and Virtual Environments, 7. 225-240. https://doi.org/10.1162/105474698565686

\section{Fabiane Friedrich Schütz}

Doutora em Psicologia pela Universidade Federal do Rio Grande do Sul (UFRGS, Porto Alegre, RS, Brasil).

\section{Livia Maria Bedin}

Doutora em Psicologia pela Universidade Federal do Rio Grande do Sul (UFRGS, Porto Alegre, RS, Brasil). Professora do Departamento de Psicologia do Desenvolvimento e da Personalidade e do Programa de Pós-Graduação em Psicologia de Psicologia da Universidade Federal do Rio Grande do Sul (UFRGS) em Porto Alegre, RS, Brasil.

\section{Jorge Castellá Sarriera}

Doutor em Psicologia pela Universidade Autonoma de Madrid (UAM, Madri, Espanha). Professor do Departamento de Psicologia do Desenvolvimento e da Personalidade e do Programa de Pós-Graduação em Psicologia e do Programa de Pós-Graduação Política Social e Serviço Social da Universidade Federal do Rio Grande do Sul (UFRGS) em Porto Alegre, RS, Brasil.

\section{Endereço para correspondência}

Fabiane Friedrich Schütz

Universidade Federal do Rio Grande do Sul - Instituto de Psicologia

Rua Ramiro Barcelos, 2600, sala 222

Santa Cecilia, 90035-003

Porto Alegre, RS, Brasil 